\title{
Effects of Sodium-Glucose Cotransporter 2 Inhibitors on Hepatic Fibrosis in Patients With Type 2 Diabetes: A Chart-Based Analysis
}

\author{
Hisayuki Katsuyama ${ }^{\text {a, b }}$, Mariko Hakoshima ${ }^{\text {a }}$, Takehiro Iijima ${ }^{\text {, }}$ \\ Hiroki Adachi ${ }^{\mathrm{a}}$, Hidekatsu Yanai ${ }^{\mathrm{a}}$
}

\begin{abstract}
Background: The presence of nonalcoholic fatty liver diseases (NAFLDs) and type 2 diabetes was associated with elevated risks of cardiovascular events as well as the progression of NAFLD to fibrosis/cirrhosis and hepatocellular carcinoma. Sodium-glucose cotransporter 2 inhibitor (SGLT2i) is a widely used antidiabetic drug, which promotes urinary excretion of glucose. Recent animal and human studies demonstrated the beneficial effects of SGLT2is on lipid accumulation and fibrosis in the liver. The purpose of the current study was to elucidate the effects of SGLT2is on hepatic fibrosis in the realworld setting.
\end{abstract}

Methods: We selected patients with type 2 diabetes who had been prescribed SGLT2is continuously for 12 months between April 1, 2014 and March 31, 2018 by a chart-based analysis. We compared the data before the SGLT2is treatment with the data at 6 and 12 months after the SGLT2is treatment started. Fibrosis in the liver was evaluated by fibrosis-4 (FIB4) index.

Results: We enrolled 315 patients in this study. The body weight, body mass index (BMI), serum levels of aspartate aminotransferase, alanine aminotransferase and $\gamma$-glutamyl transferase were significantly decreased at 6 months and maintained at 12 months, whereas there was no significant change in FIB4 index. We divided the studied patients into three groups according to the baseline FIB4 index. Only in the group of high value of the baseline FIB4 index, FIB4 index was significantly decreased at 12 months. The correlations between the change of FIB4 index during 12-month SGLT2i treatment was correlated inversely with the baseline FIB4 index.

Conclusion: Present study demonstrated that SGLT2i could ameliorate fibrosis in the liver in high-risk patients for hepatic fibrosis.

Manuscript submitted December 8, 2019, accepted January 11, 2020

aDepartment of Diabetes, Endocrinology and Metabolism, National Center for Global Health and Medicine Kohnodai Hospital, Chiba, Japan

${ }^{\mathrm{b}}$ Corresponding Author: Hisayuki Katsuyama, Department of Diabetes, Endocrinology and Metabolism, National Center for Global Health and Medicine Kohnodai Hospital, 1-7-1 Kohnodai, Ichikawa, Chiba 272-8516, Japan. Email: d-katsuyama@hospk.ncgm.go.jp

doi: https://doi.org/10.14740/jem632
Keywords: Hepatic fibrosis; Nonalcoholic fatty liver diseases; Sodium-glucose cotransporter 2 inhibitor

\section{Introduction}

Nonalcoholic fatty liver disease (NAFLD) is characterized by the presence of hepatic steatosis and ranges from simple steatosis, to nonalcoholic steatohepatitis (NASH), fibrosis/cirrhosis and hepatocellular carcinoma (HCC). The presence of type 2 diabetes (T2D) is strongly associated with NAFLD, and also relates to progression of NAFLD to NASH and HCC [1, 2]. NAFLD also increases the long-term risk of cardiovascular diseases [3].

Sodium-glucose cotransporter 2 inhibitor (SGLT2i) is a widely used antidiabetic drug which promotes urinary excretion of glucose by blocking the glucose reabsorption in the renal proximal tubules in an insulin independent manner. Recent clinical trials revealed the beneficial effects of SGLT2is on cardiovascular diseases and chronic kidney diseases $[4,5]$. We also reported that SGLT2i improved liver functions $[6,7]$. The improvement of hepatic steatosis was observed in human and animal studies, which could lead to reductions of inflammation and oxidative stress in the liver [8]. Moreover, several reports also demonstrated that SGLT2i treatment attenuated fibrosis in the liver by histological examinations or noninvasive measurements using transient elastography $[9,10]$. However, the number of studies evaluating the effects of SGLT2is on hepatic fibrosis in clinical practice is limited. Thus, we conducted present study to elucidate the effects of SGLT2is on hepatic fibrosis in the real-world setting. Hepatic fibrosis was evaluated by using noninvasive fibrosis-4 (FIB4) index, which was already reported as a useful index in NAFLD [11, 12].

\section{Materials and Methods}

This study was approval by the Institutional Ethics Committee in National Center for Global Health and Medicine (NCGMG-001910) and was also performed in accordance with the Declaration of Helsinki. We selected patients with T2D who had been prescribed SGLT2is continuously for 12 months or 
longer between April 1, 2014 and March 31, 2018 by a chartbased analysis. We compared retrospectively the data before the SGLT2is treatment with the data at 6 and 12 months after the SGLT2is treatment started. Body weight, blood pressure, plasma glucose, hemoglobin A1c (HbAlc), serum total cholesterol (TC), low-density lipoprotein-cholesterol (LDL-C), triglyceride (TG), high-density lipoprotein-cholesterol (HDLC), aspartate aminotransferase (AST), alanine aminotransferase (ALT), $\gamma$-glutamyl transferase $(\gamma \mathrm{GTP})$, creatinine as well as blood cell counts in studied subjects were measured almost at the same time point before and after 6 and 12 months treatment with SGLT2is. FIB4 index was calculated as a marker of hepatic fibrosis, using the following formula: (age $\times \mathrm{AST}) /\left(\right.$ platelet counts $\left.\left(\times 10^{9} / \mathrm{L}\right) \times \mathrm{ALT}^{1 / 2}\right)[11,13] . \mathrm{Com}-$ parison of the variables before and after the SGLT2i treatment was analyzed by a paired Student's $t$-test. Pearson's simple correlations coefficients were performed to determine the correlations between change of FIB4 index during 12-month SGLT2i treatment and various parameters. We divided the patients into three groups according to the baseline FIB4 index as follows: group 1: FIB4 index $\geq 2.67$, group 2: FIB4 index $<2.67, \geq 1.30$, group 3 : FIB4 index $<1.30$. The cutoff values were adopted from the previous reports [11]. The differences of parameters among the groups were analyzed by analysis of variance (ANOVA). All data are expressed as mean \pm standard deviation (SD). $\mathrm{P}<0.05$ was considered to be statistically significant. Statistical analysis was done using SPSS version 23 (IBM, USA).

\section{Results}

We found 356 patients who had been first prescribed SGLT2is between April 1, 2014 and March 31, 2018. We excluded 16 patients due to lack of baseline data. Twenty-five patients were also excluded since they had taken SGLT2is less than 12 months. Thus, we analyzed 315 patients in this study. Table 1 shows the baseline characteristics of the studied patients.

Changes in metabolic parameters at 6 and 12 months after the start of SGLT2is were shown in Table 2. The body weight, body mass index (BMI), plasma glucose, HbAlc, serum AST, ALT, $\gamma$ GTP, TC, HDL-C, LDL-C, TG and estimated glomerular filtration rate (eGFR) were significantly decreased, whereas hemoglobin and hematocrit were increased at 6 and 12 months after the start of SGLT2is. There was no significant change in FIB4 index.

We divided the study patients into three groups according to the baseline FIB4 index. The baseline characteristics of each group are shown in Table 3. The patients of group 1, the high-risk group for hepatic fibrosis, were older and had higher serum AST and $\gamma$ GTP levels as well as lower platelet counts.

Table 4 shows changes in clinical parameters at 6 and 12 months after the start of SGLT2is in each group. The body weight and BMI were decreased at 6 and 12 months in all groups. The serum AST levels were decreased at 6 and 12 months in groups 1 and 2. In groups 2 and 3, serum ALT levels were also decreased, and the same tendency was observed in group 1. The platelet counts were decreased at 6 and 12
Table 1. Baseline Characteristics of the Patients $(n=315)$

\begin{tabular}{ll}
\hline Age & $57.2 \pm 14.6$ \\
Sex $(\mathrm{M} / \mathrm{F})$ & $173 / 142$ \\
Body height $(\mathrm{cm})$ & $163 \pm 9$ \\
Body weight $(\mathrm{kg})$ & $77.3 \pm 18.9$ \\
BMI $\left(\mathrm{kg} / \mathrm{m}^{2}\right)$ & $29.2 \pm 6.4$ \\
Systolic blood pressure $(\mathrm{mm} \mathrm{Hg})$ & $132 \pm 18$ \\
Diastolic blood pressure $(\mathrm{mm} \mathrm{Hg})$ & $77 \pm 12$ \\
Plasma glucose (mg/dL) & $197 \pm 82$ \\
HbA1c (\%) & $8.4 \pm 1.6$ \\
SGLT2i prescribed & \\
Luseogliflozin & $116(33 \%)$ \\
Dapagliflozin & $74(23 \%)$ \\
Tofogliflozin & $39(12 \%)$ \\
Ipragliflozin & $22(7 \%)$ \\
Empagliflozin & $30(9 \%)$ \\
Canagliflozin & $34(10 \%)$ \\
\hline
\end{tabular}

BMI: body mass index; HbA1c: hemoglobin A1c; SGLT2i: sodium-glucose cotransporter 2 inhibitor.

months in group 3, but no significant change was observed in groups 1 and 2.

The changes of FIB4 index in each group are shown in Figure 1. There was no significant change in group 2. In group 1 , FIB4 index showed no significant change at 6 months, but at 12 months the decrease of FIB4 index was significant.

The correlations between the change of FIB4 index during 12-month SGLT2i treatment and various clinical parameters are shown in Table 5. The change of FIB4 index was correlated inversely with the baseline FIB4 index, but there were no significant correlations between the change of FIB4 index and the change of body weight or the change of BMI.

Table 6 shows the correlations between the change of FIB4 index during 12-month SGLT2i treatment and various clinical parameters in patients of group 1. The inverse correlation between the change of FIB4 index and the baseline FIB4 index was observed in these patients. Moreover, the same tendencies were observed between the change of FIB4 index and the change of body weight or the change of BMI.

\section{Discussion}

In this study, we examined the efficacy of the SGLT2is on hepatic fibrosis using FIB4 index in patients with T2D. The results revealed that FIB4 index was significantly decreased after 12-month SGLT2i treatment in the group with high baseline FIB4 index, whereas there was no significant change in FIB4 index in other two groups.

Previous studies revealed that SGLT2is decreased serum AST levels from the early stage after the start of SGLT2is $[6$, 7]. In the current study, the significant decrease of the serum AST levels was also observed at 6 months after the start of 
Table 2. Change of the Clinical Parameters 6 and 12 Months After the Start of SGLT2is $(n=315)$

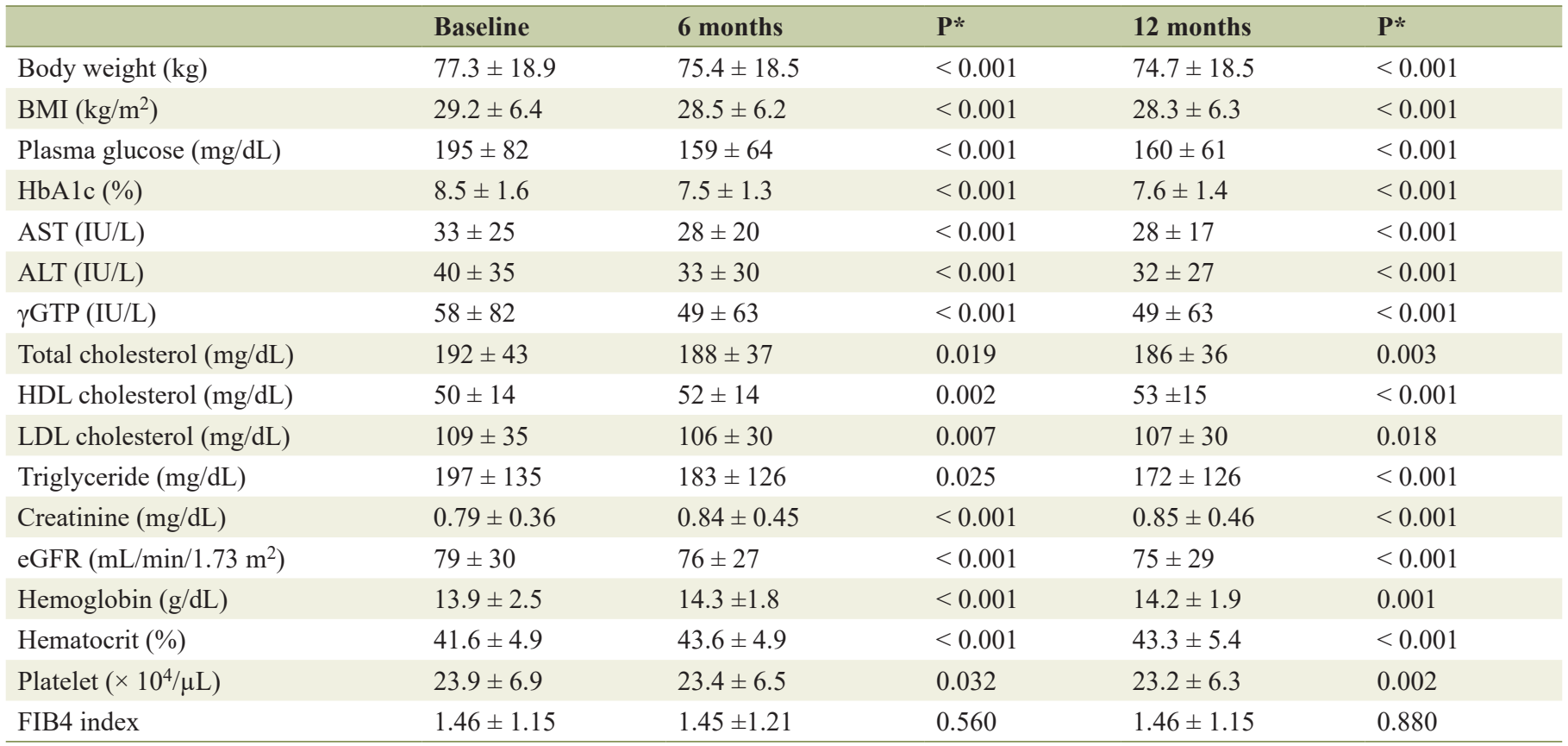

${ }^{*}$ Compared to baseline. SGLT2i: sodium-glucose cotransporter 2 inhibitor; BMI: body mass index; HbA1c: hemoglobin A1c; AST: aspartate aminotransferase; ALT: alanine aminotransferase; yGTP: y-glutamyl transferase; HDL: high-density lipoprotein; LDL: low-density lipoprotein; eGFR: estimated glomerular filtration rate; FIB4: fibrosis-4.

Table 3. Baseline Characteristics of Patients in Groups 1-3

\begin{tabular}{|c|c|c|c|c|}
\hline Group & 1 & 2 & 3 & $\mathbf{P}$ \\
\hline Baseline FIB4 index & $\geq 2.67$ & $<2.67, \geq 1.30$ & $<1.30$ & \\
\hline $\mathrm{n}(\mathrm{M} / \mathrm{F})$ & $32(17 / 15)$ & $104(56 / 48)$ & $179(100 / 79)$ & - \\
\hline Body weight (kg) & $71.7 \pm 18.2$ & $74.0 \pm 19.4$ & $80.1 \pm 18.2$ & 0.010 \\
\hline BMI $\left(\mathrm{kg} / \mathrm{m}^{2}\right)$ & $28.5 \pm 6.6$ & $27.9 \pm 5.8$ & $30.0 \pm 6.6$ & 0.038 \\
\hline Systolic blood pressure (mm Hg) & $131 \pm 16$ & $133 \pm 19$ & $131 \pm 17$ & 0.702 \\
\hline Plasma glucose $(\mathrm{mg} / \mathrm{dL})$ & $226 \pm 76$ & $191 \pm 89$ & $192 \pm 77$ & 0.116 \\
\hline HbAlc (\%) & $8.1 \pm 1.3$ & $8.5 \pm 1.8$ & $8.5 \pm 1.6$ & 0.472 \\
\hline AST (IU/L) & $54 \pm 30$ & $39 \pm 30$ & $25 \pm 15$ & $<0.001$ \\
\hline ALT (IU/L) & $45 \pm 30$ & $45 \pm 43$ & $37 \pm 30$ & 0.149 \\
\hline$\gamma \mathrm{GTP}(\mathrm{IU} / \mathrm{L})$ & $114 \pm 145$ & $68 \pm 98$ & $45 \pm 37$ & $<0.001$ \\
\hline Triglyceride $(\mathrm{mg} / \mathrm{dL})$ & $187 \pm 112$ & $188 \pm 134$ & $206 \pm 141$ & 0.507 \\
\hline Creatinine (mg/dL) & $0.83 \pm 0.22$ & $0.80 \pm 0.30$ & $0.78 \pm 0.41$ & 0.589 \\
\hline eGFR $\left(\mathrm{mL} / \mathrm{min} / 1.73 \mathrm{~m}^{2}\right)$ & $65 \pm 22$ & $74 \pm 25$ & $85 \pm 32$ & $<0.001$ \\
\hline Hemoglobin (g/dL) & $12.9 \pm 1.9$ & $13.8 \pm 1.6$ & $14.0 \pm 2.9$ & 0.055 \\
\hline Hematocrit (\%) & $39.3 \pm 5.2$ & $41.8 \pm 4.5$ & $41.9 \pm 4.9$ & 0.018 \\
\hline Platelet $\left(\times 10^{4} / \mu \mathrm{L}\right)$ & $15.1 \pm 4.9$ & $21.5 \pm 4.3$ & $26.9 \pm 6.5$ & $<0.001$ \\
\hline
\end{tabular}

FIB4: fibrosis-4; BMI: body mass index; HbA1c: hemoglobin A1c; AST: aspartate aminotransferase; ALT: alanine aminotransferase; $ү$ GTP: $\mathrm{Y}$-glutamyl transferase; HDL: high-density lipoprotein; LDL: low-density lipoprotein; eGFR: estimated glomerular filtration rate. 
Table 4. Changes of the Clinical Parameters 6 and 12 Months After the Start of SGLT2is

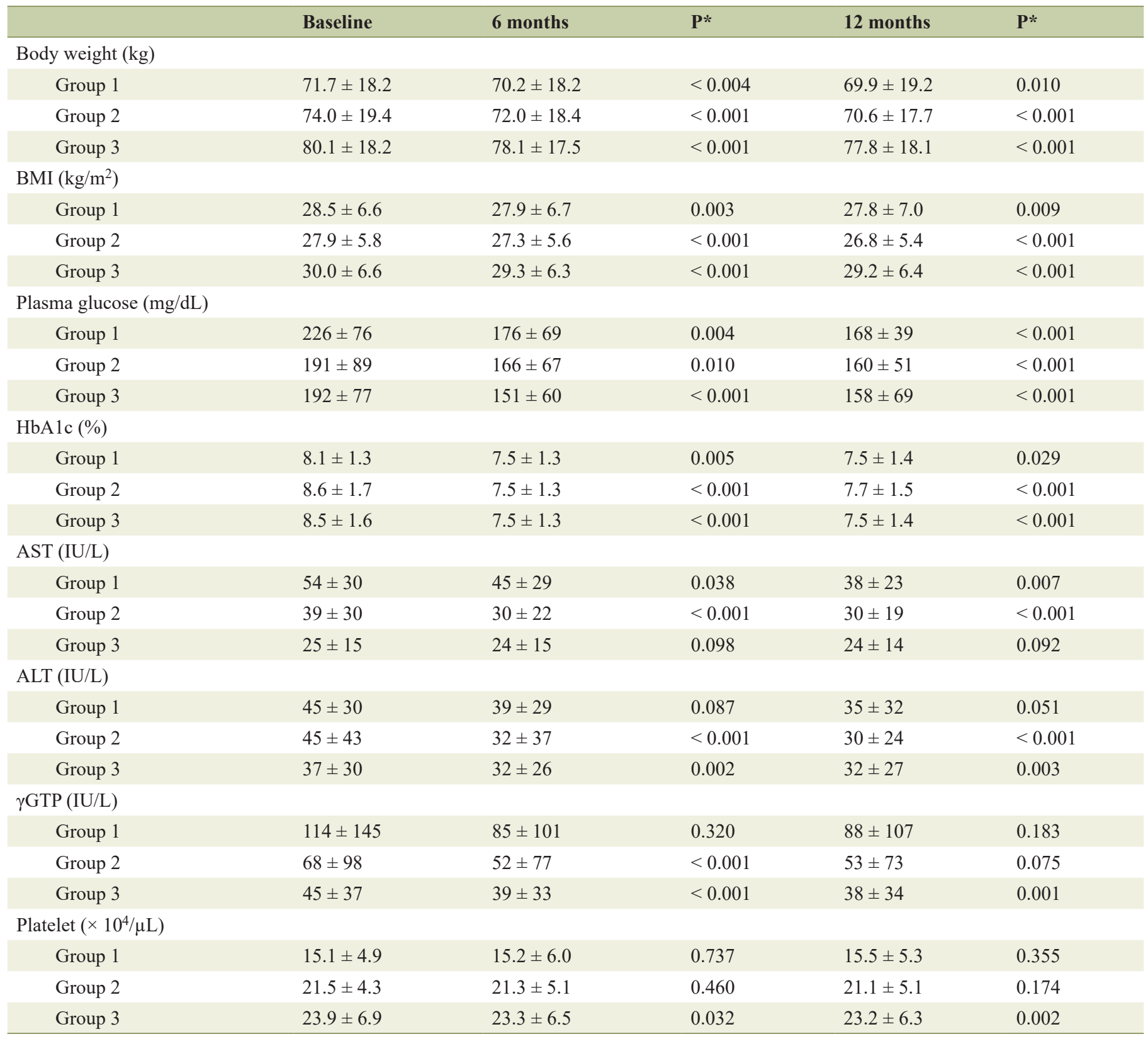

${ }^{*}$ Compared to baseline. BMI: body mass index; HbA1c: hemoglobin A1c; AST: aspartate aminotransferase; ALT: alanine aminotransferase; yGTP: Y-glutamyl transferase.

SGLT2i treatment, and was maintained at 12 months. Several mechanisms in the improvement of liver injury were suggested [8]. Obata et al reported that tofogliflozin decreased hepatic fat content and expression levels of lipogenic genes in high fat diet fed mice [14]. In this study, insulin resistance was ameliorated by increasing glucose uptake in skeletal muscle and lipolysis in adipose tissue [14]. In high-fat diet and streptozotocin-nicotinamide-induced T2D mice, ipragliflozin improved hepatic steatosis as well as reduced plasma and liver levels of oxidative stress biomarkers and inflammatory markers [15]. The improvement in hepatic steatosis after SGLT2i treatment was also reported in humans. Kahl et al clearly demonstrated by a randomized controlled study that the 24-week empagliflozin treatment in well-controlled T2D patients effectively lowered hepatic fat content assessed by magnetic resonance imaging, and increased insulin sensitivity as well as adiponectin levels [16]. The reduction of hepatic fat content was also reported after the treatment with dapagliflozin and canagliflozin in patients with T2D $[17,18]$. A recent study demonstrated the reduction of hepatic fat content after the 24-week luseogliflozin treatment in Japanese patients with T2D and NAFLD [19]. SGLT2is could activate adenosine 5'-monophosphate-activat- 


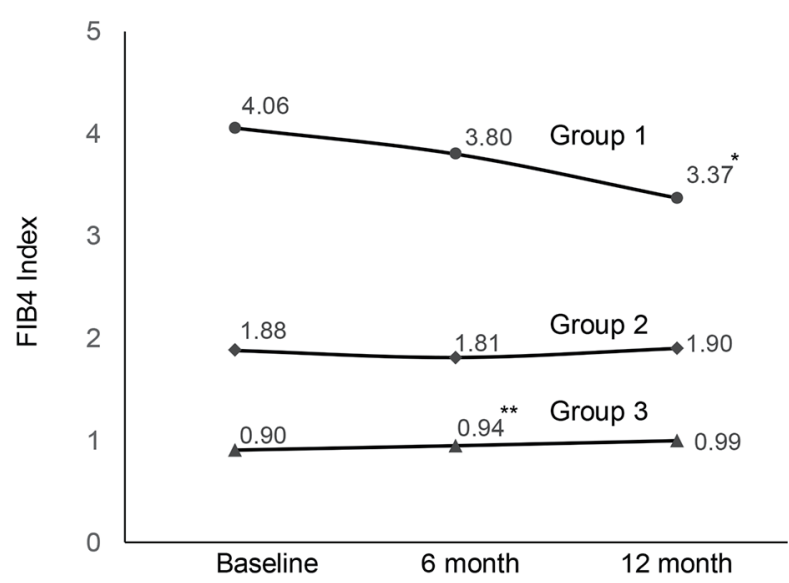

Figure 1. Changes in FIB4 index during SGLT2i treatment $\left({ }^{*} P=0.003\right.$, $\left.{ }^{* *} \mathrm{P}=0.005\right)$. FIB4: fibrosis-4; SGLT2i: sodium-glucose cotransporter 2 inhibitor.

ed protein kinase (AMPK) by inhibiting mitochondrial function and elevating adiponectin levels [16, 20], leading to upregulation of fatty acid (FA) oxidation in skeletal muscle and liver as well as downregulation of FA synthesis in the liver [21, $22]$. The decrease of fat accumulation reduces oxidative stress and inflammation [8].

We used FIB4 index as a marker of fibrosis in the liver. FIB4 index was first developed as a simple noninvasive index to predict hepatic fibrosis in patients with hepatitis $\mathrm{C}$ virus and human immunodeficiency virus co-infection [13]. Shah et al

Table 5. Correlations Between Changes of FIB4 Index During 12-Month SGLT2i Treatment and Clinical Parameters

\begin{tabular}{lll}
\hline & R & P \\
\hline Age & 0.012 & 0.828 \\
Baseline body weight & -0.097 & 0.290 \\
Change of body weight & 0.014 & 0.809 \\
Baseline BMI & -0.064 & 0.269 \\
Change of BMI & -0.005 & 0.927 \\
Baseline HbA1c & 0.028 & 0.626 \\
Chang of HbA1c & -0.002 & 0.977 \\
Baseline AST & -0.300 & $<0.001$ \\
Change of AST & 0.454 & $<0.001$ \\
Baseline ALT & -0.128 & 0.023 \\
Change of ALT & -0.154 & 0.006 \\
Baseline $\gamma$ GTP & -0.187 & 0.003 \\
Change of $\gamma$ GTP & 0.348 & $<0.001$ \\
Baseline platelet & 0.139 & 0.014 \\
Change of platelet & -0.175 & 0.002 \\
Baseline FIB4 index & -0.375 & $<0.001$ \\
\hline
\end{tabular}

FIB4: fibrosis-4; SGLT2i: sodium-glucose cotransporter 2 inhibitor; BMI: body mass index; HbA1c: hemoglobin A1c; AST: aspartate aminotransferase; ALT: alanine aminotransferase; yGTP: y-glutamyl transferase.
Table 6. Correlations Between Changes of FIB4 Index During 12-Month SGLT2i Treatment and Clinical Parameters in Patients of Group 1

\begin{tabular}{lll}
\hline & R & P \\
\hline Age & 0.105 & 0.567 \\
Baseline body weight & 0.034 & 0.860 \\
Change of body weight & -0.325 & 0.085 \\
Baseline BMI & 0.081 & 0.676 \\
Change of BMI & -0.312 & 0.099 \\
Baseline HbA1c & 0.145 & 0.452 \\
Chang of HbA1c & -0.010 & 0.960 \\
Baseline AST & -0.420 & 0.017 \\
Change of AST & 0.646 & $<0.001$ \\
Baseline ALT & -0.133 & 0.467 \\
Change of ALT & 0.513 & 0.003 \\
Baseline $\gamma \mathrm{GTP}$ & -0.061 & 0.774 \\
Change of $\gamma \mathrm{GTP}$ & 0.319 & 0.121 \\
Baseline platelet & -0.028 & 0.880 \\
Change of platelet & -0.533 & 0.002 \\
Baseline FIB4 index & -0.510 & 0.003 \\
\hline
\end{tabular}

FIB4: fibrosis-4; SGLT2i: sodium-glucose cotransporter 2 inhibitor; BMI: body mass index; HbA1c: hemoglobin A1c; AST: aspartate aminotransferase; ALT: alanine aminotransferase; yGTP: y-glutamyl transferase.

demonstrated that FIB4 index was superior for the diagnosis of advanced fibrosis in NAFLD, compared to other six indexes [11]. The utility of FIB4 index was also confirmed in Japanese patients with NAFLD [12]. There was a possibility that the values of FIB4 index may be influenced by age because of its formula [23]. Indeed, in the current study, there was a significant difference in age among three groups, which were divided according to the baseline FIB4 index. However, we analyzed the changes of FIB4 index during SGLT2i treatment in each patient. The influences of age on the current results were considered to be limited.

In the current study, FIB4 index was significantly decreased after 12-month SGLT2i treatment in the group with high value of the baseline FIB4 index, suggesting that SGLT2is may improve fibrosis in the liver in patients with advanced fibrosis. Several reports showed that SGLT2i treatment reduced scores of fibrosis as well as steatosis and ballooning by histological examinations [9, 24]. Shimizu et al assessed fibrosis in the liver by measuring liver stiffness with the transient elastography and reported that a significant improvement of liver stiffness after 24-week dapagliflozin was only observed in the patients with high degree of baseline liver stiffness [10]. This result agreed with our results. Several studies already evaluated changes in FIB4 index during the SGLT2i treatment [25]. Nevertheless, only one study showed significant reduction in FIB4 index after ipragliflozin administration [26], and most studies failed to show significant change in FIB4 index [20, 27]. The short duration of SGLT2i treatment as well as the degree of hepatic fibrosis in studied patients might influence the 
result. In NASH model mice, canagliflozin inhibited the development of hepatic fibrosis and reduced the number of liver tumors, suggesting that SGLT2is could attenuate or delay the progression of NAFLD to NASH and HCC [28]. The underlying mechanisms of the effects of SGLT2is in hepatic fibrosis are still unclear. In the group with high value of baseline FIB4 index in our study, the change of FIB4 index was tended to be inversely correlated with the change of body weight, suggesting that the weight reduction might contribute to inhibiting the progression of hepatic fibrosis.

The present study has several limitations. First, we analyzed the results of six kinds of SGLT2is together, which were available in Japan, and did not consider the possible differences among SGLT2is. However, it has been thought that SGLT2 is have similar effects because of its similarity in the chemical structure. Second, we could not exclude possible influences from other treatments and life habits, such as hypoglycemic, anti-hypertensive, or lipid lowering agents, food intakes and/ or exercise levels. Third, since this study was based on retrospective chart analysis, there was lack of data, which might influence the result.

Despite these limitations, this study provided the realworld data about the efficacy of SGLT2 is on improving fibrosis in the liver, which could support insights obtained from previous studies. Well-designed prospective clinical studies are required to elucidate the mechanisms of the improvements of the effects of SGLT2is on fibrosis in the liver.

\section{Conclusion}

The present study demonstrated that SGLT2i could ameliorate fibrosis in the liver in high-risk patients for hepatic fibrosis in the real-world setting.

\section{Acknowledgments}

We thank Harue Aoki and Ayano Sakakibara of the Division of Research Support, National Center for Global Health and Medicine Kohnodai Hospital, for their great support in data collection.

\section{Financial Disclosure}

Authors have no financial disclosures to report.

\section{Conflict of Interest}

The authors declare that they have no conflict of interest concerning this article.

\section{Informed Consent}

Not applicable.

\section{Author Contributions}

HK designed the research. HK, MH, TI, HA and HY collected data. HK, MH, TI and HY analyzed data. HK and HY wrote the paper. All authors read and approved the final paper.

\section{References}

1. Portillo-Sanchez P, Bril F, Maximos M, Lomonaco R, Biernacki D, Orsak B, Subbarayan S, et al. High prevalence of nonalcoholic fatty liver disease in patients with type 2 diabetes mellitus and normal plasma aminotransferase levels. J Clin Endocrinol Metab. 2015;100(6):2231-2238.

2. Adams LA, Lymp JF, St Sauver J, Sanderson SO, Lindor KD, Feldstein A, Angulo P. The natural history of nonalcoholic fatty liver disease: a population-based cohort study. Gastroenterology. 2005;129(1):113-121.

3. Targher G, Bertolini L, Rodella S, Tessari R, Zenari L, Lippi G, Arcaro G. Nonalcoholic fatty liver disease is independently associated with an increased incidence of cardiovascular events in type 2 diabetic patients. Diabetes Care. 2007;30(8):2119-2121.

4. Zinman B, Wanner C, Lachin JM, Fitchett D, Bluhmki E, Hantel S, Mattheus M, et al. Empagliflozin, cardiovascular outcomes, and mortality in type 2 diabetes. N Engl J Med. 2015;373(22):2117-2128.

5. Perkovic V, Jardine MJ, Neal B, Bompoint S, Heerspink HJL, Charytan DM, Edwards R, et al. Canagliflozin and renal outcomes in type 2 diabetes and nephropathy. N Engl J Med. 2019;380(24):2295-2306.

6. Katsuyama H, Hamasaki H, Adachi H, Moriyama S, Kawaguchi A, Sako A, Mishima S, et al. Effects of sodium-glucose cotransporter 2 inhibitors on metabolic parameters in patients with type 2 diabetes: a chart-based analysis. J Clin Med Res. 2016;8(3):237-243.

7. Yanai H, Hakoshima M, Adachi H, Kawaguchi A, Waragai Y, Harigae T, Masui Y, et al. Effects of six kinds of sodium-glucose cotransporter 2 inhibitors on metabolic parameters, and summarized effect and its correlations with baseline data. J Clin Med Res. 2017;9(7):605-612.

8. Yanai H, Hakoshima M, Katsuyama H. The possible mechanisms for improvement of liver function due to sodium-glucose cotransporter-2 inhibitors. J Clin Med Res. 2019;11(11):769-772.

9. Lai LL, Vethakkan SR, Nik Mustapha NR, Mahadeva S, Chan WK. Empagliflozin for the Treatment of Nonalcoholic Steatohepatitis in Patients with Type 2 Diabetes Mellitus. Dig Dis Sci. 2019.

10. Shimizu M, Suzuki K, Kato K, Jojima T, Iijima T, Murohisa T, Iijima M, et al. Evaluation of the effects of dapagliflozin, a sodium-glucose co-transporter-2 inhibitor, on hepatic steatosis and fibrosis using transient elastography in patients with type 2 diabetes and non-alcoholic fatty liver disease. Diabetes Obes Metab. 2019;21(2):285-292.

11. Shah AG, Lydecker A, Murray K, Tetri BN, Contos MJ, Sanyal AJ, Nash Clinical Research N. Comparison of noninvasive markers of fibrosis in patients with nonal- 
coholic fatty liver disease. Clin Gastroenterol Hepatol. 2009;7(10):1104-1112.

12. Sumida Y, Yoneda M, Hyogo H, Itoh Y, Ono M, Fujii H, Eguchi Y, et al. Validation of the FIB4 index in a Japanese nonalcoholic fatty liver disease population. BMC Gastroenterol. 2012;12:2.

13. Sterling RK, Lissen E, Clumeck N, Sola R, Correa MC, Montaner J, M SS, et al. Development of a simple noninvasive index to predict significant fibrosis in patients with HIV/HCV coinfection. Hepatology. 2006;43(6):13171325.

14. Obata A, Kubota N, Kubota T, Iwamoto M, Sato H, Sakurai Y, Takamoto I, et al. Tofogliflozin Improves Insulin Resistance in Skeletal Muscle and Accelerates Lipolysis in Adipose Tissue in Male Mice. Endocrinology. 2016;157(3):1029-1042.

15. Tahara A, Kurosaki E, Yokono M, Yamajuku D, Kihara R, Hayashizaki Y, Takasu T, et al. Effects of SGLT2 selective inhibitor ipragliflozin on hyperglycemia, hyperlipidemia, hepatic steatosis, oxidative stress, inflammation, and obesity in type 2 diabetic mice. Eur J Pharmacol. 2013;715(1-3):246-255.

16. Kahl S, Gancheva S, Strassburger K, Herder C, Machann J, Katsuyama H, Kabisch S, et al. Empagliflozin effectively lowers liver fat content in well-controlled type 2 diabetes: a randomized, double-blind, phase 4, placebocontrolled trial. Diabetes Care. 2020;43(2):298-305.

17. Eriksson JW, Lundkvist P, Jansson PA, Johansson L, Kvarnstrom M, Moris L, Miliotis T, et al. Effects of dapagliflozin and n-3 carboxylic acids on non-alcoholic fatty liver disease in people with type 2 diabetes: a doubleblind randomised placebo-controlled study. Diabetologia. 2018;61(9):1923-1934.

18. Cusi K, Bril F, Barb D, Polidori D, Sha S, Ghosh A, Farrell K, et al. Effect of canagliflozin treatment on hepatic triglyceride content and glucose metabolism in patients with type 2 diabetes. Diabetes Obes Metab. 2018.

19. Sumida Y, Murotani K, Saito M, Tamasawa A, Osonoi Y, Yoneda M, Osonoi T. Effect of luseogliflozin on hepatic fat content in type 2 diabetes patients with non-alcoholic fatty liver disease: A prospective, single-arm trial (LEAD trial). Hepatol Res. 2019;49(1):64-71.

20. Hawley SA, Ford RJ, Smith BK, Gowans GJ, Mancini SJ, Pitt RD, Day EA, et al. The $\mathrm{Na}+/$ Glucose cotransporter inhibitor canagliflozin activates AMPK by inhibiting mi- tochondrial function and increasing cellular AMP levels. Diabetes. 2016;65(9):2784-2794.

21. Chiang H, Lee JC, Huang HC, Huang H, Liu HK, Huang C. Delayed intervention with a novel SGLT2 inhibitor NGI001 suppresses diet-induced metabolic dysfunction and non-alcoholic fatty liver disease in mice. Br J Pharmacol. 2019.

22. Xu L, Nagata N, Nagashimada M, Zhuge F, Ni Y, Chen G, Mayoux E, et al. SGLT2 inhibition by empagliflozin promotes fat utilization and browning and attenuates inflammation and insulin resistance by polarizing M2 macrophages in diet-induced obese mice. EBioMedicine. 2017;20:137-149.

23. Ishiba H, Sumida Y, Tanaka S, Yoneda M, Hyogo H, Ono M, Fujii H, et al. The novel cutoff points for the FIB4 index categorized by age increase the diagnostic accuracy in NAFLD: a multi-center study. J Gastroenterol. 2018;53(11):1216-1224.

24. Akuta N, Kawamura Y, Watanabe C, Nishimura A, Okubo M, Mori Y, Fujiyama S, et al. Impact of sodium glucose cotransporter 2 inhibitor on histological features and glucose metabolism of non-alcoholic fatty liver disease complicated by diabetes mellitus. Hepatol Res. 2019;49(5):531-539.

25. Scheen AJ. Effect of sodium-glucose cotransporter type 2 inhibitors on liver fat in patients with type 2 diabetes: hepatic beyond cardiovascular and renal protection? Ann Transl Med. 2018;6(Suppl 1):S68.

26. Ito D, Shimizu S, Inoue K, Saito D, Yanagisawa M, Inukai K, Akiyama Y, et al. Comparison of ipragliflozin and pioglitazone effects on nonalcoholic fatty liver disease in patients with type 2 diabetes: a randomized, 24week, open-label, active-controlled trial. Diabetes Care. 2017;40(10):1364-1372.

27. Kurinami N, Sugiyama S, Yoshida A, Hieshima K, Miyamoto F, Kajiwara K, Jinnouch K, et al. Dapagliflozin significantly reduced liver fat accumulation associated with a decrease in abdominal subcutaneous fat in patients with inadequately controlled type 2 diabetes mellitus. Diabetes Res Clin Pract. 2018;142:254-263.

28. Shiba K, Tsuchiya K, Komiya C, Miyachi Y, Mori K, Shimazu N, Yamaguchi S, et al. Canagliflozin, an SGLT2 inhibitor, attenuates the development of hepatocellular carcinoma in a mouse model of human NASH. Sci Rep. 2018;8(1):2362. 